\title{
Does anomalous color vision imply color weakness?'
}

\section{Leo M. Hurvich and Dorothea Jameson UNIVERSITY OF PENNSYLANIA}

\begin{abstract}
"Reduction" explanations of anomalous color vision imply that deuteranomalous systems are green-weak and protanomalous red-weak. An experimental test fails to confirm this interpretation, but is consistent with an alternative explanation that postulates opponent-response systems for color anomalies that are shifted relative to those of normal color vision.
\end{abstract}

\section{Introduction}

The commonest form of aberrant color vision is anomalous trichromatism. Observers with such vision require, like the normal, three physical stimuli to match colors of the visible spectrum, but they require them in different amounts or proportions than the normal observer (Engelking, 1927). The most generally held view of the anomalous trichromats is that they are color weak and represent forms intermediate between normal and dichromatic vision.

A single color equation (or minor variants of it) has long been used to diagnose color anomalies. The equation has the form:

$$
\mathrm{a}(535 \mathrm{~m} \mu)+\mathrm{b}(670 \dot{\mathrm{m}} \mu)=\mathrm{c}(589 \mathrm{~m} \mu)
$$

This is the Rayleigh equation, although Rayleigh did not specify the wavelengths. Observers (deuteranomalous) who require an excess of an $535 \mathrm{~m} \mu$ (yellowish-green) when mixed with $670 \mathrm{~m} \mu$ (yellowish-red) to match $589 \mathrm{~m} \mu$ (largely yellow) are typically tagged "'green weak." Similarly, those (protanomalous) who require an excess of the $670 \mathrm{~m} \mu$, when mixed with $535 \mathrm{~m} \mu$, are tagged "red weak."

\section{Color theory}

The facts of anomalous trichromatism pose serious difficulties for color theory. According to traditional Young-Helmholtz three-component analysis, the explanation usually (but not always) given for such vision is that "it is a reduction system in which the stimulus values for any light of one of the components is uniformly less than normal" (Parsons, 1924). The concept of simple reduction, however, implies that the same color equations are acceptable to both normal and anomalous observers (Jameson and Hurvich,

\footnotetext{
1 Abridgement of a paper read at International Congress of Optics, Munieh, August, 1962
}

1956) which is contradictory to the fact that normal and anomalous color equations do differ. To overcome this specific difficulty, Pitt (1949) assumed that for protanomaly; the long wave distribution function undergoes progressive logarithmic reductions and that each degree of modification of this function is associated with a certain degree of reduction in red sensitivity.

The opponent-process theory of color vision provides a different explanation of anomalous trichromatism. Two basic assumptions are made: (1) that in such abnormalities the spectral sensitivities of the normal photopigments are shifted to different degrees as a group along the frequency ( $1 /$ wavelength) scale, thereby causing systematic alterations in the paired chromatic and achromatic response systems; and (2) in addition, there may be progressive degrees of reduction or loss of function of the red-green chromatic response pair (Jameson and Hurvich, 1956).

One rather simple consequence of the alternative theoretical views can be put to direct experimental test by determining the position in the spectrum of the unique yellow point of anomalous observers. According to Pitt's logarithmic "reduction" analysis of Young-Helmholtz theory, unique yellow should be displaced toward the red for the protanomalous. This is because yellow, in the theory, is determined by the intersection of red and green curves, and a reduction

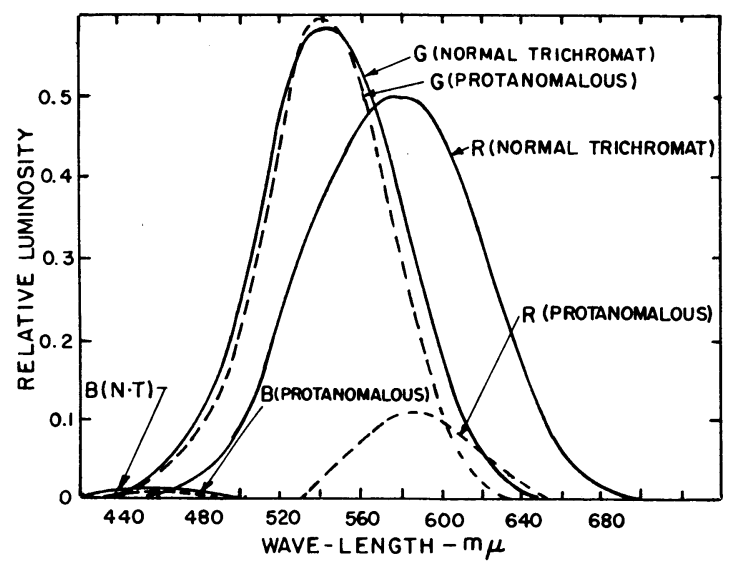

Fig. 1. "Fundamental response curves"' (equal energy spectrum) for normal trichromat and protanomalous observer. After Pitt (1949). 
of the red-sensitivity curve would cause the intersection to move toward the long wavelengths (see Fig. 1). On the other hand, opponent-colors theory predicts that the shift of unique yellow in protanomalous observers should be toward the green or short wavelengths. Shifts in the opposite directions would be anticipated for deuteranomaly in the two views.

\section{Results}

The direct experimental check of the opposite alternative predictions is shown in Fig. 2. The figure shows on the ordinate the spectral locus of unique yellow of 49 observers ranging in age from 16 to 51 years. This locus was determined for a condition of neutral adaptation (Hurvich and Jameson, 1951) against the midpoint value of each observer's Rayleigh equation (542 $\mathrm{m} \mu$ and $670 \mathrm{~m} \mu=592 \mathrm{~m} \mu)$; measured with a Nagel anomaloscope (Willis and Farnsworth, 1952).

The Rayleigh match scores (20-28) for the 6 deuteranomalous observers are lower than the normals and their unique yellow loci (590$597 \mathrm{m \mu}$ ) are at longer wavelengths than those of the normal group. The three protanomalous observers have Rayleigh match scores (66-73)

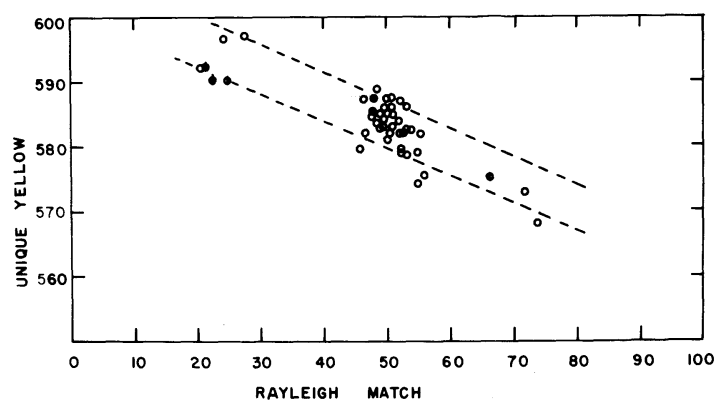

Fig. 2. Rayleigh equation mid-point scores and spectral loci of unique yellow for 49 observers: 40 normals, 6 deuteranomalous and 3 protanomalous.

higher than those of the normal group and their unique yellow loci (568-575 $\mathrm{m} \mu)$ are displaced toward the shorter wavelengths. The data make it clear that the deuteranomalous observers, who use more of the yellowish-green stimulus in the Rayleigh match and who are described by the "reduction" hypothesis as green-weak, nevertheless, see the normal unique yellow stimulus as greenish-yellow and they select as pure yellow a stimulus of longer wavelength which is seen by the normal observer as orange. The protanomalous observers, who use more of the yellowish-red stimulus in the Rayleigh match and who are described by the "reduction" hypothesis as red-weak, see the normal unique yellow as orange, and select as their pure yellow a wavelength that looks greenish to the normal observer. It is clear that the deviant color systems of anomalous observers do not necessarily imply specific red or green color weaknesses.

\section{References}

ENGELKING, E. Ueber den Verlauf der Eichwertkurven bei den anomalen Trichromaten. Klin. Monatsbl. Augenheilk., 1927, 78 (Suppl.), 209-219.

HURVICH, L. M. and JAMESON, D. The binocular fusion of yellow in relation to color theories.Science, 1951, 114, 199-202.

JAMESON, D. and HURVICH, L. M. Theoretical analysis of anomalous trichromatic color vision. J. Opt. Soc. Amer., 1956, 46, 1075-1089.

- PARSONS, J. H. An Introduction to the Study of Colour Vision. Cambridge: Cambridge University Press, 1924. P. 229.

PITT, F. H. G. Some aspects of anomalous vision. Documenta Ophthal., 1949, 3, 307-317.

WILLIS, M. P. and FARNSWORTH, D. Comparative evaluation of anomaloscopes. Med. Res. Lab. Rep. No. $190,1952,11$, No. $7,1-89$.

\section{Acknowled gment}

The research project of which this study forms a part was supported by Grant B 1721 from the National Institutes of Health. The Nagel anomaloscope used in the study was kindly loaned to us by Dr. Hans-Lukas Teuber of the Massachusetts Institute of Technology. Miss Irene Levine served as research assistant and collected most of the experimental data. 